\title{
EECDA: Energy Efficient Clustering and Data Aggregation Protocol for Heterogeneous Wireless Sensor Networks
}

\author{
D. Kumar, T.C. Aseri, R.B. Patel
}

\author{
Dilip Kumar \\ Centre for Development of Advanced Computing, Mohali, India \\ E-mail: dilip.k78@gmail.com
}

Trilok C. Aseri

PEC University of Technology, Chandigarh, India

E-mail: a_trilok_chand@yahoo.com

R.B. Patel

M.M. University, Mullana, India

E-mail: patel_r_b@yahoo.com

\begin{abstract}
In recent years, energy efficiency and data gathering is a major concern in many applications of Wireless Sensor Networks (WSNs). One of the important issues in WSNs is how to save the energy consumption for prolonging the network lifetime. For this purpose, many novel innovative techniques are required to improve the energy efficiency and lifetime of the network. In this paper, we propose a novel Energy Efficient Clustering and Data Aggregation (EECDA) protocol for the heterogeneous WSNs which combines the ideas of energy efficient cluster based routing and data aggregation to achieve a better performance in terms of lifetime and stability. EECDA protocol includes a novel cluster head election technique and a path would be selected with maximum sum of energy residues for data transmission instead of the path with minimum energy consumption. Simulation results show that EECDA balances the energy consumption and prolongs the network lifetime by a factor of $51 \%$, $35 \%$ and $10 \%$ when compared with Low-Energy Adaptive Clustering Hierarchy (LEACH), Energy Efficient Hierarchical Clustering Algorithm (EEHCA) and Effective Data Gathering Algorithm (EDGA), respectively.

Keywords: clustering; data aggregation; lifetime; heterogeneous wireless sensor networks.
\end{abstract}

\section{Introduction}

For past few years, Wireless Sensor Networks (WSNs) attracted lots of researchers because of its potential wide applications and many research challenges. Early study on WSNs mainly focused on technologies based on the homogeneous WSN in which all nodes have same system resources. However, heterogeneous WSN is becoming more and more popular because the benefits of using heterogeneous WSNs with different capabilities in order to meet the demands of various applications have been presented in recent literature [1], [2].

One of the crucial challenges in the organization of the WSNs is energy efficiency and stability because battery capacities of sensor nodes are limited and replacing them are impractical. Since, sensor nodes use a large amount of energy for data transmission and aggregation. Therefore, new energy efficient routing protocols are required to save energy consumption. In this paper, we propose a novel Energy-Efficient Clustering and Data Aggregation (EECDA) protocol for heterogeneous WSN. In this approach, a new Cluster Head $(\mathrm{CH})$ election and data communication mechanism is presented to extend the lifetime and stability of the network. After the 
CHs election, a path with maximum sum of residual energy would be selected for data communication instead of the path with minimum energy consumption. Therefore, each $\mathrm{CH}$ first aggregates the received data and then transmits the aggregated data to the Base Station (BS). The main contributions of EECDA protocol is to provide longest stability (when the first node is dead) and improves the network lifetime in comparison to Low-Energy Adaptive Clustering Hierarchy (LEACH), Energy-Efficient Hierarchical Clustering Algorithm (EEHCA) and Effective Data Gathering Algorithm (EDGA).

The rest of this paper is organized as follows. Section 2 presents related works. Section 3 describes the EECDA protocol. Section 4 explores on simulation results, and finally paper is concluded in Section 5.

\section{Related Work}

Many recent research works in the area of cluster-based WSNs have extensively focussed on energy efficiency, lifetime, stability and scalability. In the past few years, numerous clustering algorithms have been proposed for a wide range of applications [3], [4], [5].

Data aggregation and hierarchical mechanism are commonly used in many critical applications of WSNs. It reduces the data redundancy and communication load [6]. LEACH [7] is the first clustering protocol based on single-hop communication model. In LEACH, during the setup phase, each node generates a random number between 0 and 1 . If this random number is smaller than the threshold value, $T(s)$, which is given by Equation (1), then the node becomes a $\mathrm{CH}$ for the current round. During each round, new CHs are elected and as a result balanced load energy is distributed among the CHs and other nodes of the network.

$$
T(s)=\left(\begin{array}{cc}
\frac{p_{\text {opt }}}{1-p_{\text {opt }} \times\left(r \bmod \frac{1}{p_{\text {opt }}}\right)} & \text { if } s \in G \\
0 & \text { otherwise }
\end{array}\right)
$$

where $p_{\text {opt }}$ is the desired percentage of CHs, $r$ is the count of current round, $G$ is the set of sensor nodes that have not been CHs in the last $\frac{1}{p_{o p t}}$ rounds. In this paper, we refer round, $\frac{1}{p_{o p t}}$ , as epoch of the heterogeneous WSN.

Power-Efficient Gathering in Sensor Information Systems (PEGASIS) [8] is a chain-based power efficient protocol based on LEACH. It assumes that each node must know the location of all other nodes. It starts with the farthest node and the chain is constructed by using a greedy algorithm. The chain leader aggregates data and forwards it to the BS. In order to balance the overhead involved in communication between the chain leader and the BS, each node in the chain takes turn to be the leader. In [9], the authors described a heuristic approach to solve the data-gathering problem with aggregation in sensor networks. In this scheme, the data is collected in an efficient manner from all the sensor nodes and transmitted to the BS to maximize the lifetime of the network.

In [10], the authors have studied the impact of heterogeneity of sensor nodes in terms of their energy and proposed a heterogeneous-aware protocol to prolong the time interval before the death of the first node. In [11] a cost-based comparative study between homogeneous and heterogeneous clustered WSNs is proposed to estimate the optimal distribution among different types of sensors, but this result is hard to use if the heterogeneity is due to the operation of the network. In [12], authors have developed energy efficient clustering protocol in WSN which is more suitable for periodical data gathering applications. A survey on many ad hoc and mobile ad hoc network clustering schemes are presented in [13]. In this article authors observed that new clustering schemes are required to handle the topology maintenance and managing node 
movement in the network. In [14], the authors have proposed a new data gathering approach for single-hop transmission wherein both the data gathering and the aggregation are performed by the same sensor in a cluster but the report to the BS may be done by a different sensor.

In [15], authors have investigated the problem of cluster formation for data fusion by focusing on two aspects: (i) how does one can estimate the number of clusters needed to utilize efficiently data correlation of sensors for a sensor network, and (ii), how does one can pick the CHs to cover the whole network more efficiently. In [16], the authors have analyzed the strengths and weaknesses of many existing clustering algorithms and observed many solutions of appropriate aggregation metrics those have been recently proposed in the literature. Energy-Efficient Protocol with Static Clustering (EEPSC) which partitions the network into static clusters and utilizes CHs to distribute the energy load among high power sensor nodes and extends the network lifetime [17].

A distributed energy saving clustering algorithm called BPEC has been proposed in [18]. In this algorithm, CHs are selected by two probabilities. First is based on the ratio between average residual energy of neighbor nodes and its residual energy and second is the node's degree. By using this algorithm, the entire network broadcasting complexity is $O(n)$, the entire network computing complexity is $O(1)$. The results show that when the network has a higher communication coverage density, analytical and experimental results are very close. Energy-Efficient Hierarchical Clustering Algorithm (EEHCA) [19] has adopted a new method for $\mathrm{CH}$ election, which can avoid the frequent election of CHs. A new concept of backup CHs is introduced which improves the performance over LEACH and Hybrid Energy-Efficient Distributed clustering (HEED), in terms of network lifetime. An energy efficient hierarchical data gathering protocol, called EDGA adopts weighted election probabilities of each heterogeneous sensor node to become a $\mathrm{CH}$ which better handle heterogeneous energy circumstances [20]. The results demonstrate that EDGA significantly outperforms LEACH and HEED in terms of network lifetime.

The authors in [21] have discussed a new $\mathrm{CH}$ election problem based on a set of coverage-aware cost metrics which favor nodes deployed in densely populated network areas. The coverage-aware election of CH nodes, active sensor nodes and routers in clustered WSN increases the lifetime as compared with traditional energy based election methods. In [22], the authors have presented an important corona model to maximize the network lifetime by using maximal transmission range of sensors into different levels. The sensor nodes belong to the same corona have the same transmission range, whereas different coronas have different transmission ranges. In [23] authors have presented a short survey on the main techniques used for energy conservation in WSNs. The main focus is primarily on duty cycle scheme which represents the most suitable technique for energy saving. In [24], the authors reviewed many existing definitions of network lifetime and discussed about the merits and demerits of these definitions.

\section{EECDA Protocol}

The main goal of EECDA protocol is to maintain efficiently the energy consumption of sensor nodes by involving them in a single-hop communication within a cluster. The data aggregation and fusion technique is used to reduce the number of transmitted messages to the BS to save the energy and prevent the congestion. To make the protocol implementation, we have adopted a few reasonable assumptions as follows: (i) $n$ sensor nodes are uniformly dispersed within a square field; (ii) All sensor nodes and the BS are stationary after deployment; (iii) The WSN consists of heterogeneous nodes in terms of node energy; (iv) CHs perform data aggregation; (v) The BS is not energy limited in comparison with the energy of other nodes in the network.

We use the same radio model defined in [7]. The amount of energy required to transmit a $L$ bit packet over a distance, $d$, is given by Equation (2). 


$$
E_{T x}(L, d)=\left(\begin{array}{cc}
L \times E_{\text {elec }}+L \times \epsilon_{f s} \times d^{2} & \text { if } d<=d_{0} \\
L \times E_{\text {elec }}+L \times \epsilon_{m p} \times d^{4} & \text { if } d>=d_{0}
\end{array}\right)
$$

$E_{\text {elec }}$ is the energy being dissipated to run the transmitter or receiver circuitry. The parameters $\epsilon_{m p}$ and $\epsilon_{f s}$ is the amount of energy dissipates per bit in the radio frequency amplifier according to the distance $d_{0}$, which is given by Equation (3).

$$
d_{0}=\sqrt{\frac{\epsilon_{f s}}{\epsilon_{m p}}}
$$

The amount of energy required to receive a packet is given by Equation (4).

$$
E_{R x}(L)=L \times E_{\text {elec }}
$$

\subsection{Impacts of heterogeneity on network performance}

By placing few heterogeneous nodes in the network can bring three main benefits: (i) Extending network lifetime: the average energy consumption for forwarding a packet from the heterogeneous nodes to a BS will be much less than the energy consumed in homogeneous sensor networks, (ii) Improving reliability of data communication: the heterogeneous sensor network can get much higher end-to-end delivery rate than the homogeneous sensor network and (iii) Decreasing latency of data transmission: the heterogeneous nodes can decrease the forwarding latency by using fewer hops to the BS.

\subsection{Optimal number of clusters}

The optimal probability of a node to become a $\mathrm{CH}$ is very important in WSNs. This clustering is optimal in the sense that energy consumption is well distributed among all the sensor nodes and the total energy consumption should be minimum. Such optimal clustering highly depends on the energy model. For EECDA, we have used similar energy model as discussed in [7]. Let us assume an area $A=M \times M$ square meters over which n nodes are uniformly distributed. For simplicity, assume the BS is located in the center of the field, and the distance of any node to the $\mathrm{BS}$ or its $\mathrm{CH}$ is $d_{0}$. Therefore, the energy dissipated by the $\mathrm{CH}$ node during a round is given by the Equation (5).

$$
E_{C H}=\left(\frac{n}{k}\right) \times L \times\left(E_{\text {elec }}+E_{D A}\right)+L \times \epsilon_{f s} \times d_{B S}^{2}
$$

where $k$ is the number of clusters, $E_{D A}$ is the data aggregation and $d_{B S}$ is the average distance between a $\mathrm{CH}$ and the $\mathrm{BS}$ which is given by Equation (6).

$$
d_{B S}^{2}=\int \sqrt{\left(x^{2}+y^{2}\right)} \times \frac{1}{A}=0.765 \times \frac{M}{2}
$$

The energy dissipated by a non- $\mathrm{CH}$ node is given by Equation (7).

$$
E_{N C H}=L \times\left(E_{\text {elec }}+\epsilon_{f s} \times d_{C H}^{2}\right)
$$

where $d_{C H}$ is the average distance between a non- $\mathrm{CH}$ node and its associated $\mathrm{CH}$, which is given by Equation (8) [10].

$$
d_{C H}^{2}=\iint\left(x^{2}+y^{2}\right) \times \rho(x, y) d x d y=\frac{M^{2}}{2 \pi k}
$$


where $\rho(x, y)$ is the node distribution and $M$ is the area of monitoring field. The total energy dissipated in a cluster per round is given by Equation (9).

$$
E_{T}=E_{C H}+E_{N C H}
$$

By substituting Equation (5) and Equation (7) in Equation (9), we obtain energy dissipating during a round which is given by Equation (10).

$$
E_{T}=L \times\left(2 \times n \times E_{\text {elec }}+n \times E_{D A}+\epsilon_{f s} \times\left(k \times d_{B S}^{2}+n \times \frac{M^{2}}{2 \pi k}\right)\right.
$$

By setting the derivative $E_{T}$ with respect to $k$ to zero, we derive the optimal number of clusters which is given by Equation (11).

$$
k_{\text {opt }}=\sqrt{\frac{n}{2 \pi}} \times \sqrt{\frac{\epsilon_{f s}}{\epsilon_{m p}}} \times \frac{M}{d_{B S}^{2}}
$$

Using Equation (6) and Equation (11), the optimal probability of a node to become a $\mathrm{CH}, p_{\text {opt }}$, can be computed by Equation (12)

$$
p_{\text {opt }}=\frac{1}{0.765} \times \sqrt{\frac{2}{n \pi}} \times \sqrt{\frac{\epsilon_{f s}}{\epsilon_{m p}}}
$$

If the clusters are not constructed in an optimal way, the total energy dissipated per round is increased exponentially either when the number of clusters is greater or less than the optimal value.

\subsection{CH election phase}

EECDA considers three types of nodes (i.e., normal, advanced and super) which have deployed in a harsh wireless environment where battery replacement is impossible. Nodes with higher battery energy are advanced and super nodes and the remaining nodes are normal nodes. The main aim of EECDA is to increase the energy efficiency, lifetime and stability of the network in the presence of heterogeneous nodes. Let $m$ be the fraction of advanced nodes among the normal nodes and $\left(m_{o}\right)$ be the proportion of super nodes among the advanced nodes. Let us assume the initial energy of each normal node is $E_{0}$. The initial energy of each advanced and super node is $E_{0} \times(1+\alpha)$ and $E_{0} \times(1+\beta)$, where both $\alpha$ and $\beta$ means the advanced and super node have $\alpha$ and $\beta$ times more energy than the normal node. Intuitively, advanced and super nodes have to become CHs more often than the normal nodes, which is equivalent to a fairness constraint on energy consumption. The new heterogeneous setting has no affect on the spatial density of the network so the priori setting of, $p_{\text {opt }}$, does not change but the total energy of the network will be changed. The total initial energy of the new heterogeneous network setting is given by Equation (13).

$$
n \times E_{0} \times\left\{(1-m)+m \times\left(\left(1-m_{o}\right) \times(1+\alpha)+m_{o} \times(1+\beta)\right\}\right)=n \times E_{0} \times\left(1+m \times\left(\alpha-m_{o} \times(\alpha-\beta)\right)\right)
$$

The first improvement to the existing protocols is to increase the epoch of the sensor network in proportion to the energy increment. In order to optimize the stable region of the system, the new epoch must become equal to $\left(\frac{1}{p_{\text {opt }}}\right) \times\left(1+m \times\left(\alpha-m_{o} \times(\alpha-\beta)\right)\right)$ because the system has $m \times\left(\alpha-m_{o} \times(\alpha-\beta)\right)$ times more energy due to heterogeneous nodes. 
If we set the same threshold value for super, advanced and normal nodes with the difference that each normal node $\epsilon G$ becomes a $\mathrm{CH}$ once every $\left(\frac{1}{p_{\text {opt }}}\right) \times\left(1+m \times\left(\alpha-m_{o} \times(\alpha-\beta)\right)\right)$ rounds per epoch, and each advanced and super node $\epsilon G$ becomes a $\mathrm{CH}(1+\alpha)$ and $(1+\beta)$ times every $\left(\frac{1}{p_{\text {opt }}}\right) \times\left(1+m \times\left(\alpha-m_{o} \times(\alpha-\beta)\right)\right)$ rounds per epoch, then there is no guarantee that the number of CHs per round per epoch will be $p_{\text {opt }} \times n$. This problem can be overcome by modifying the threshold Equation (1).

In EECDA, we assign a weight to the optimal probability $p_{\text {opt }}$. This weight must be equal to the initial energy of each node divided by the initial energy of the normal node. Let us define $p_{n}, p_{a}$, and $p_{s}$ are the weighted election probabilities for normal, advanced and super nodes. Virtually there are $n \times\left(1+m \times\left(\alpha-m_{o} \times(\alpha-\beta)\right)\right)$ nodes with energy equal to the initial energy of a normal node. In order to maintain the minimum energy consumption in each round within an epoch, the average number of CHs per round per epoch must be constant and equal to $p_{\text {opt }} \times n$. In the heterogeneous scenario, the average number of CHs per round per epoch is equal to $\left(1+m \times\left(\alpha-m_{o} \times(\alpha-\beta)\right)\right) \times n \times p_{n}$ because each virual node has the initial energy of a normal node. Therefore, the weighed probabilities for normal, advanced and super nodes are respectively given by Equations (14-16).

$$
\begin{gathered}
p_{n}=\frac{p_{\text {opt }}}{\left(1+m \times\left(\alpha-m_{o} \times(\alpha-\beta)\right)\right.} \\
p_{a}=\frac{p_{\text {opt }}}{\left(1+m \times\left(\alpha-m_{o} \times(\alpha-\beta)\right)\right.} \times(1+\alpha) \\
p_{s}=\frac{p_{o p t}}{\left(1+m \times\left(\alpha-m_{o} \times(\alpha-\beta)\right)\right.} \times(1+\beta)
\end{gathered}
$$

By substituting Equation (14) in Equation (1) and a new threshold is derived for normal nodes which is given by Equation (17).

$$
T\left(s_{n}\right)=\left(\begin{array}{cc}
\frac{p_{n}}{1-p_{n} \times\left(r \bmod \frac{1}{p_{n}}\right)} & \text { if } s_{n} \in G^{\prime} \\
0 & \text { otherwise }
\end{array}\right)
$$

where $r$ is the current round, $G^{\prime}$ is the set of normal nodes that have not become $\mathrm{CHs}$ within the last, $\frac{1}{p_{n}}$, rounds of the epoch and $T(s n)$ is the threshold applied to a population of $n \times(1-m)$ normal nodes. This guarantees that each normal node will become a $\mathrm{CH}$ exactly once every $\left(\frac{1}{p_{\text {opt }}}\right) \times\left(1+m \times\left(\alpha-m_{o} \times(\alpha-\beta)\right)\right)$ rounds per epoch, and that the average number of $\mathrm{CHs}$ of normal nodes per round per epoch is equal to $\left(n \times(1-m) \times p_{n}\right)$. Similarly, new thresholds for advanced and super nodes can be derived by substituting Equation (15) and (16) into Equation (1), which are given by Equation (18) and Equation (19).

$$
\begin{aligned}
T\left(s_{a}\right) & =\left(\begin{array}{cc}
\frac{p_{a}}{1-p_{a} \times\left(r \bmod \frac{1}{p_{a}}\right)} & \text { if } s_{a} \in G^{\prime \prime} \\
0 & \text { otherwise }
\end{array}\right) \\
T\left(s_{s}\right) & =\left(\begin{array}{cc}
\frac{p_{s}}{1-p_{s} \times\left(r \bmod \frac{1}{p_{s}}\right)} & \text { if } s_{s} \in G^{\prime \prime \prime} \\
0 & \text { otherwise }
\end{array}\right)
\end{aligned}
$$




\section{Route selection phase}

Once all $\mathrm{CHs}$ are elected in a specific round by using weighted election probability, each $\mathrm{CH}$ first estimates its energy residue $E_{(\mathrm{CHR})_{s}}$ and broadcast this information with its $\mathrm{CH}$ role to the neighboring nodes. The value of $E_{(C H R)_{s}}$ can be calculated by Equation (20).

$$
E_{(C H R)_{s}}=\left(E_{(C H r e m)_{s}}-\left(E_{(B S)_{s}}\right) \quad s \in G_{c}\right.
$$

where $G_{c}$ is the set of elected CHs per round. $\left(E_{(\mathrm{CHrem})_{s}}\right.$ indicates the remaining energy of $\mathrm{CH}_{s}$ in current round and $\left(\mathrm{E}_{(B S)_{s}}\right)$ indicates the communication energy dissipated from $\mathrm{CH}_{s}$ to the BS. Then, each $\mathrm{CH}$ records the value of $\left(E_{(\mathrm{CHR})_{s}}\right)$ in advertisement message and broadcasts advertisement message to the rest of the nodes in the WSN. During the $\mathrm{CH}$ election phase, each non- $\mathrm{CH}$ node receives all advertisement messages, and extracts all of energy residue data of $\mathrm{CH}_{s}$ from advertisement messages.

Moreover, each non-CH node also calculates energy residues $\left(E_{(\mathrm{NCHR})_{i}}\right)$ to every $\mathrm{CH}$ respectively which is given by Equation (21).

$$
E_{(N C H R)_{i}}=\left(E_{(\mathrm{NCHrem})_{i}}-\left(E_{(\mathrm{CH})_{i s}}\right) \quad i \epsilon G_{n}\right.
$$

where $G_{n}$ is the set of non-CH nodes. $\left(E_{\left(\mathrm{NCHrem}_{i}\right.}\right)$ indicates the residual energy of non-CH node $i$ in the current round and $\left(E_{(C H)_{i s}}\right)$ indicates the communication energy from non- $\mathrm{CH}$ node $i$ to $\mathrm{CH}$ node s. Finally, each non- $\mathrm{CH}$ node would associate one of the existing $\mathrm{CH}$ according to maximum energy residue which is given by Equation (22). Therfore, a path with a maximum sum of energy residues would be selected for data transmission in spite of that path with minimum energy consumption.

$$
\operatorname{Max}\left\{E_{(C H R)_{s}}+E_{(N C H R)_{i}}\right\} \quad s \in G_{c}, i \in G_{n}
$$

\section{Data communication phase}

In data communication phase, each non- $\mathrm{CH}$ node transmits its data to the associated $\mathrm{CH}$. Each $\mathrm{CH}$ will receive all sensed data from its associated non-CH nodes and sends it to the BS.

\section{Simulation Results and Discussion}

To evaluate and compare the performance of EECDA with EEHCA, EDGA and LEACH in the heterogeneous WSN, we have conducted simulations for two scenarios: first, a network with 100 nodes deployed over an area of size $100 \times 100$ square meter, and second, a network with 200 nodes deployed over an area of size $200 \times 200$ square meter as shown in Figure 1 and Figure 2 , we denote a normal node with (o), an advanced node with $(+)$, a super node with $\left(^{*}\right)$ and the BS with (x). The simulation parameters are summarized in Table 1.

The performance metrics used for these protocols are: (i) Network Lifetime: this is the time interval from the start of the operation until the first and last node dies; (ii) Stability Period: this is the time interval from the start of the operation until the death of the first alive node; (iii) Instability Period: this is the time interval from the death of the first alive node until the death of the last alive node and (iv) Number of alive nodes per round: this is the instantaneous measure reflects that the total number of alive nodes per round that have not yet expended all of their energy.

Figure 3 and Figure 4 show that both LEACH and EEHCA fails to take the full advantage of heterogeneity in both the scenarios where the first and last node dies earlier as compared to EDGA and EECDA. Therefore, EECDA prolongs the network lifetime by $51 \%, 35 \%$ and $10 \%$ 


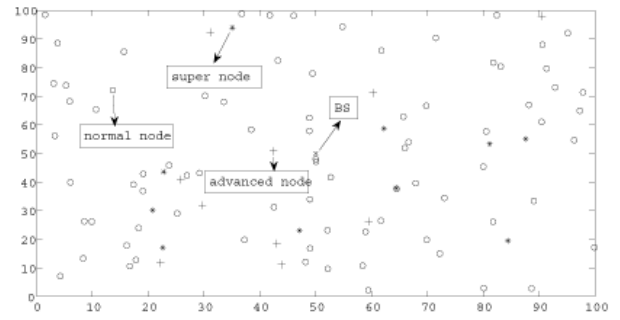

Figure 1: Random deployment of 100 nodes over an area $100 \times 100 \mathrm{~m}^{2}$.

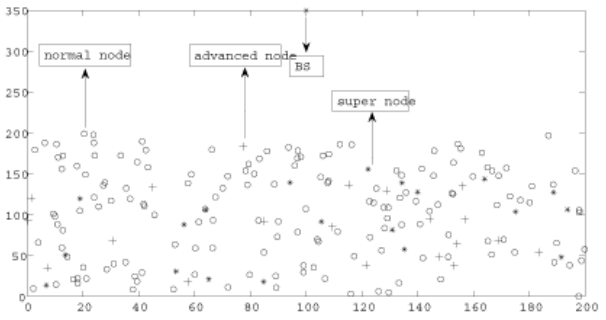

Figure 2: Random deployment of 200 nodes over an area $200 \times 200 \mathrm{~m}^{2}$.

Table 1: Simulation parameters

\begin{tabular}{|c|c|}
\hline Parameter & Scenario I and II \\
\hline Network area & $100 \times 100 \mathrm{~m}^{2}, 200 \times 200 \mathrm{~m}^{2}$ \\
\hline BS location & $(50,50),(100,350)$ \\
\hline$n$ & 100,200 \\
\hline$E_{D A}$ & $5 n \mathrm{~J} / \mathrm{bit} / \mathrm{report}$ \\
\hline Packet size & $50 \mathrm{bytes}$ \\
\hline$\epsilon_{m p}$ & $0.0013 \mathrm{pJ} / \mathrm{bit} / \mathrm{m}^{4}$ \\
\hline$\epsilon_{f s}$ & $10 \mathrm{pJ} / \mathrm{bit} / \mathrm{m}^{2}$ \\
\hline$E_{\text {elec }}$ & $50 \mathrm{~nJ} / \mathrm{bit}$ \\
\hline
\end{tabular}

when compared with LEACH, EEHCA and EDGA protocols, respectively. Figures 3, 4, 5 and 6 present that the unstable region for EECDA is shorter than that of LEACH, EEHCA and EDGA because the normal nodes die in both the scenarios very fast in case of LEACH, EEHCA and EDGA that result in the sensing field it become sparse very fast. On the other hand, advanced and super nodes die in a very slow fashion, because they are not selected as CHs very often after the death of the normal nodes, which again affects the election process of CHs and makes the network unstable. It is quite evident that the stable region of EECDA is extended as compared with LEACH, EEHCA and EDGA for both the scenarios. Figure 5 and Figure 6 indicate that the number of alive nodes are more per round in case of EECDA as compared with EDGA, EEHCA and LEACH because a path with a maximum sum of energy residual would be selected for data transmission in spite of that path with minimum energy in case of EECDA.

Figures 7, 8, 9, 10, 11 and 12 illustrate the performance of residual energy of normal, advanced and super nodes under the heterogeneous settings of EECDA, EDGA, EEHCA and LEACH. Initially, EECDA has the same initial energy as EDGA, LEACH and EEHCA, but gradually it decreases in EDGA, EEHCA and LEACH over rounds. So, EDGA, EEHCA and LEACH have less residual energy left after certain rounds for both the scenarios. Therefore, more the residual energy more efficient is the system.

\section{Conclusion}

Most existing research considers homogeneous sensor networks. However, a homogeneous sensor network suffers from poor performance and scalability. In this paper, we have developed 
EECDA: Energy Efficient Clustering and Data Aggregation Protocol for Heterogeneous

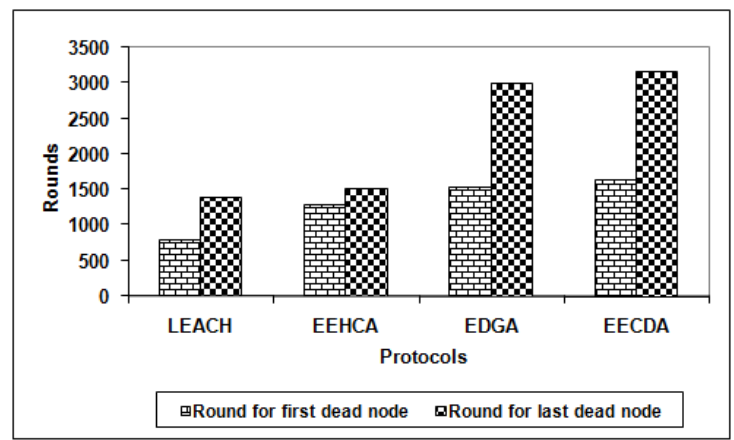

Figure 3: Network lifetime as a function of first and last dead nodes over an area $100 \times 100 \mathrm{~m}^{2}$.

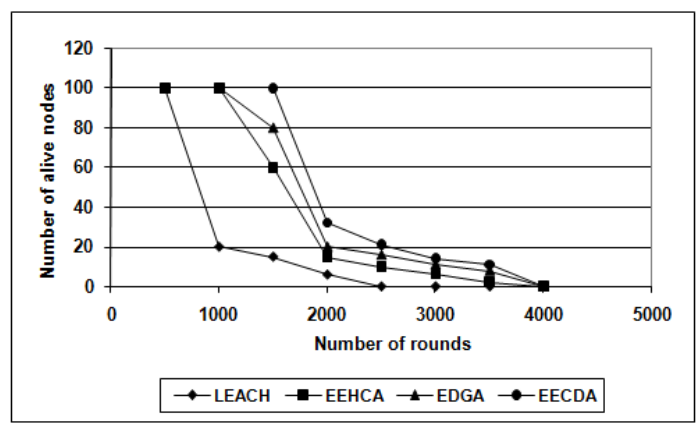

Figure 5: Stability as a function of number of alive nodes per round over an area $100 \times 100 \mathrm{~m}^{2}$.

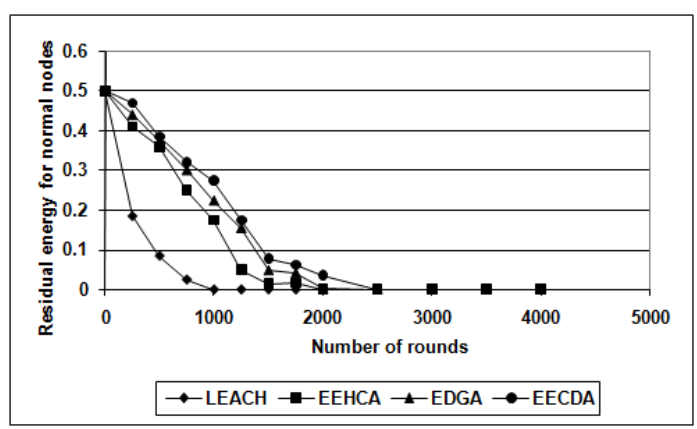

Figure 7: Residual energy of normal nodes per round over an area $100 \times 100 \mathrm{~m}^{2}$.

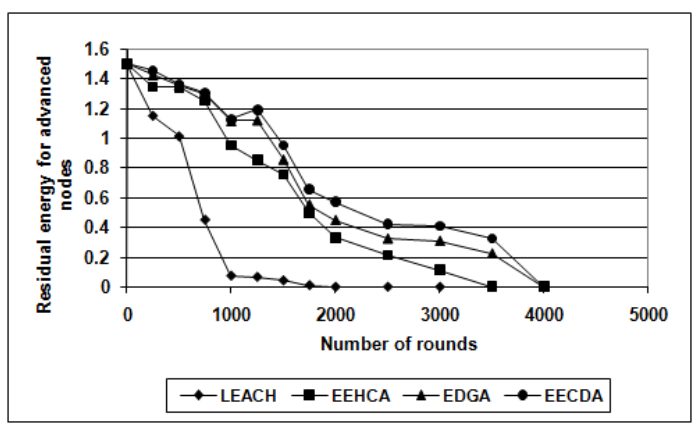

Figure 9: Residual energy of advanced nodes per round overan area $100 \times 100 \mathrm{~m}^{2}$.

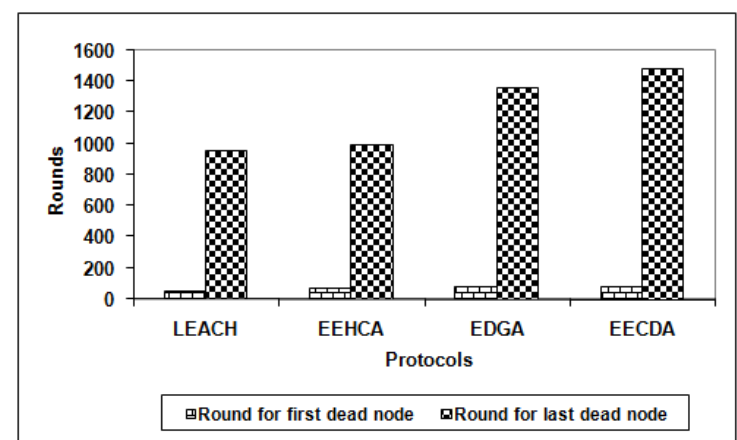

Figure 4: Network lifetime as a function of first and last dead nodes over an area $200 \times 200 \mathrm{~m}^{2}$.

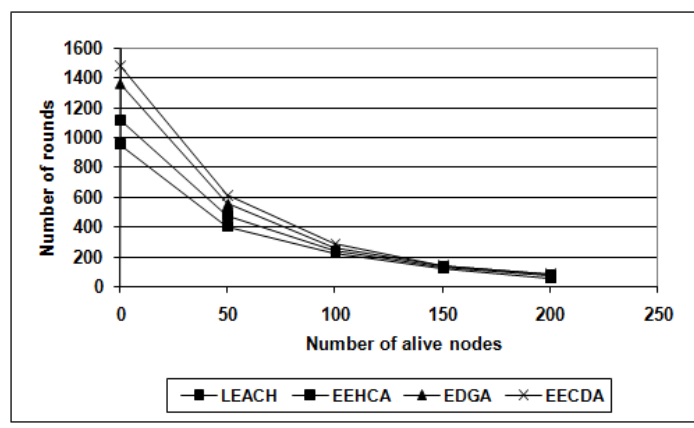

Figure 6: Stability as a function of number of alive nodes per round over an area $200 \times 200 \mathrm{~m}^{2}$.

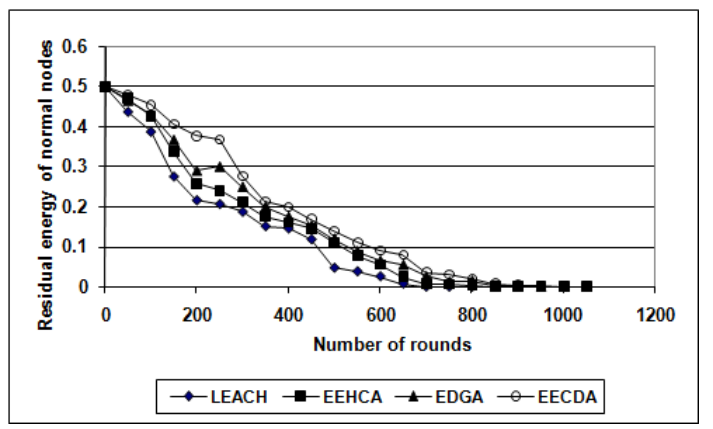

Figure 8: Residual energy of normal nodes per round over an area $200 \times 200 \mathrm{~m}^{2}$.

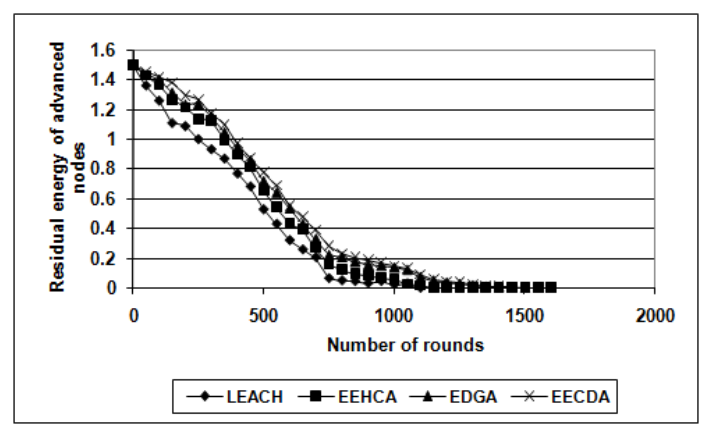

Figure 10: Residual energy of advanced nodes per round over an area $200 \times 200 \mathrm{~m}^{2}$. 


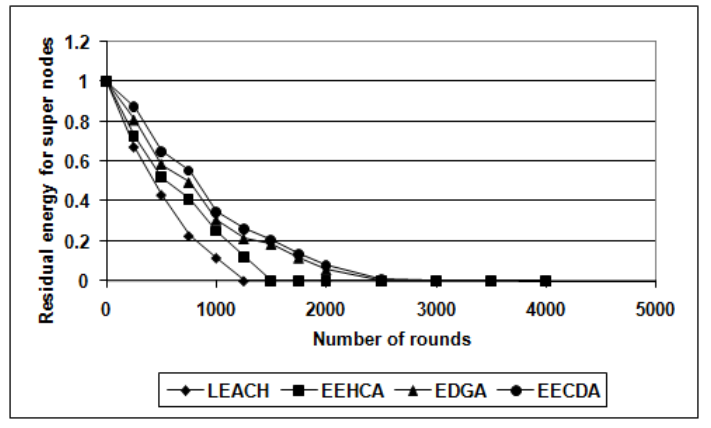

Figure 11: Residual energy of super nodes per round overan area $100 \times 100 \mathrm{~m}^{2}$.

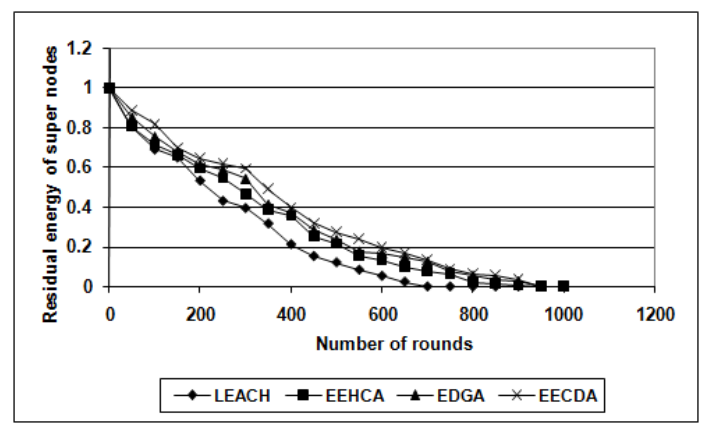

Figure 12: Residual energy of super nodes per round over an area $200 \times 200 \mathrm{~m}^{2}$.

a novel Energy Efficient Clustering and Data Aggregation (EECDA) protocol to improve the network performance by using some heterogeneous nodes in the network. A novel cluster head election technique and a path with maximum sum of energy residual for data transmission can maintain the balance of energy consumption in the network. Simulation results show that EECDA has better network lifetime, stability and energy efficiency when compared with EDGA, EEHCA and LEACH protocols. The future work includes more levels of hierarchy with some mobility in the network.

\section{Bibliography}

[1] R Kumar, V. Tsiatsis, M.B. Srivastava, Computation Hierarchy for In-Network Processing, Proceedings of 2nd ACM International Workshop on Wireless Networks and Applications, San Diego, CA, 68-77, 2003.

[2] D. Kumar, T. C. Aseri, R.B. Patel, HCEE: Hierarchical clustered energy efficient protocol for heterogeneous wireless sensor networks, International Journal of Electronics Engineering, 1(1):123-126, 2009.

[3] J. Yu, P. Chong, A Survey of Clustering Schemes for Mobile Ad Hoc Networks, IEEE Communication Surveys,7(1): 32-48, 2005.

[4] N. Vlajic, D. Xia, Wireless Sensor Networks: To Cluster or Not to Cluster?, Proceedings of International Symposium on a Wireless of Wireless, Mobileand Multimedia Networks (WoWMoM'06), 259-268, 2006.

[5] W.S. Jang, W.M. Heley, M.J. Skibniewsk, Wireless Sensor Networks as a Part of Web Based Building Environment Monitoring System, Automation in Construction Journal, 17: 729-736, 2008.

[6] B. Krishnamachari, D. Estrin, S.B. Wicker, The Impact of Data Aggregation in Wireless Sensor Networks, Proceedings of 22nd International Conference on Distributed Computing Systems Workshops (ICDCSW'02), 575-578, 2002.

[7] W.B. Heinzelman, A.P. Chandrakasan, H. Balakrishnan, An Application-Specific Protocol Architecture for Wireless Microsensor Networks, IEEE Transactions on Wireless Communications, 1(4):660-669, 2002.

[8] S. Lindesy, C. Raghavendra, PEGASIS: Power-Efficient Gathering in Sensor Information System, Proceedings of IEEE Aerospace Conference, 1-6, 2002. 
[9] K. Dasgupta, K. Kalpakis, P. Namjoshi, An Efficient Clustering-Based Heuristic for Data Gathering and Aggregation in Sensor Networks, Proceedings of Wireless Communication and Networking (WCNC'03), 3: 1948-1953, 2003.

[10] G. Smaragdakis, I. Matta, A. Bestavros, SEP: A Stable Election Protocol For Clustered Heterogeneous Wireless Sensor Networks, Proceedings of 2nd International Workshop on Sensor and Actor Network Protocols and Applications (SANPA'04),Boston, MA, 660-670, 2004.

[11] V. Mhatre, C. Rosenberg, Homogeneous vs. Heterogeneous Clustered Sensor Networks: A Comparative Study, Proceedings of IEEE International Conference on Communications (ICC'04), 2004.

[12] M. Ye, C. Li, G. Chen, J. Wu, EECS: An Energy Efficient Clustering Scheme in Wireless Sensor Networks, Proceedings of 24th IEEE International Performance, Computing and Communications Conference (IPCCC'05), 535- 540, 2005.

[13] D. Wei, H. A. Chan, Clustering Ad Hoc Networks: Schemes and Classifications, Proceedings of 3rd Annual IEEE Communication Society on Sensors and Ad hoc Communication and Networks (SECON'06),, 3: 920-926, 2006.

[14] H. Yuning, Z. Yongbing, J. Yusheng, S. Xuemin, A New Energy Efficient Approach by Separating Data Collection and Data Report in Wireless Sensor Networks, Proceedings of International Conference on Communication and Mobile Computing (IWCMC'06), 1165-1170, 2006.

[15] H. Chen, S. Megerian, Cluster Sizing and Head Selection for Efficient Data Aggregation and Routing in Sensor Networks, Proceedings of Wireless Communication and Networking (WCNC'06), 4: 2318-2323,2006.

[16] S. Xun, A Combinatorial Algorithmic Approach To Energy Efficient Information Collection In Wireless Sensor Networks, ACM Transactions on Sensor Networks, 3(1), 2007.

[17] A. S. Zahmati, B. Abolhassani, A. A. B. Shirazi, A. S. Bakhtiari, An Energy-Efficient Protocol with Static Clustering for Wireless Sensor Networks, International Journal of Electronics, Circuits and Systems, 3(2): 135-138, 2007.

[18] X. Jianbo, H. Yong, L. I. Renfa, An Energy-Aware Distributed Clustering Algorithm in Wireless Sensor Networks, Proceedings of International Conference on Computer Science and Software Engineering, 528-531, 2008.

[19] G. Xin, W.H. Yang, D. DeGang, EEHCA: An Energy-Efficient clustering Algorithm for Wireless Sensor Networks, Information Technology Journal, 7(2):245-252, 2008.

[20] Y. Mao, Z. Liu, L. Zhang, X. Li, An Effective Data Gathering Scheme in Heterogeneous Energy Wireless Sensor Networks, Proceedings of International Conference on Computational Science and Engineering, 338-343, 2009.

[21] A. Bari, A. Jaekel, S. Bandyopadhyay, Clustering Strategies for Improving the Lifetime of Two-Tiered Sensor Networks, Elsevier, Computer Communication Journal, 31(14): 3451$3459,2008$.

[22] C. Song, M. Liu, J. Cao, Y. Zheng et. al, Maximizing Network Lifetime Based on Transmission Range Adjustment in Wireless Sensor Networks, Elsevier, Computer Communication Journal , 32(11): 1316-1325, July 2009. 
[23] H.Y. Shiue, J.X Lieo-hong, S. Horijuchi, Energy Saving in Wireless Sensor Networks, Journal of Communication and Computing , 6(5):20-28, 2009.

[24] I. Dietrich, F. Dressler, On the Lifetime of Wireless Sensor Networks, ACM Transactions on Sensor Networks, 5(1): 5:1-5:39, 2009. 